\title{
Near-threshold quantization and level densities for potential wells with weak inverse-square tails
}

\author{
Michael J. Moritz, Christopher Eltschka, and Harald Friedrich \\ Physik-Department, Technische Universität München, 85747 Garching, Germany
}

(Received 13 March 2001; published 6 July 2001)

\begin{abstract}
For potential tails consisting of an inverse-square term and an additional attractive $1 / r^{m}$ term, $V(r)$ $\sim\left[\hbar^{2} /(2 \mathcal{M})\right]\left[\left(\gamma / r^{2}\right)-\left(\beta^{m-2} / r^{m}\right)\right]$, we derive the near-threshold quantization rule $n=n(E)$ which is related to the level density via $\rho=d n / d E$. For a weak inverse-square term, $-\frac{1}{4}<\gamma<\frac{3}{4}$ (and $m>2$ ), the leading contributions to $n(E)$ are $n=A-B(-E)^{\sqrt{\gamma+1 / 4}}$, so $\rho$ has a singular contribution proportional to $(-E)^{\sqrt{\gamma+1 / 4}-1}$ near threshold. The constant $B$ in the near-threshold quantization rule also determines the strength of the leading contribution to the transmission probability through the potential tail at small positive energies. For $\gamma=0$ we recover results derived previously for potential tails falling off faster than $1 / r^{2}$. The weak inverse-square tails bridge the gap between the more strongly repulsive tails, $\gamma \geqslant 3 / 4$, where $n(E) \stackrel{E \rightarrow 0}{=} A+O(E)$ and $\rho$ remains finite at threshold, and the strongly attractive tails, $\gamma<-1 / 4$, where

$n \stackrel{E \rightarrow 0}{=} B \ln (-E / A)$, which corresponds to an infinite dipole series of bound states and connects to the behavior $n \stackrel{E \rightarrow 0}{=} A+B E^{(1 / 2)-(1 / m)}$, describing infinite Rydberg-like series in potentials with longer-ranged attractive tails falling off as $1 / r^{m}, 0<m<2$. For $\gamma=-1 / 4$ (and $m>2$ ) we obtain $n(E) \stackrel{E \rightarrow 0}{=} A+C / \ln (-E / B$ ), which remains finite at threshold.
\end{abstract}

DOI: 10.1103/PhysRevA.64.022101

PACS number(s): 03.65.Ge, 03.65.Sq, 03.65.Xp

\section{INTRODUCTION}

Recent intense activity related to cold atoms and their interactions has led to increased interest in threshold properties of atomic and molecular potentials. One property of interest is the level density $\rho(E)$, which is closely related to the quantization rule,

$$
n=n(E), \quad \rho(E)=\frac{d n}{d E} .
$$

The quantization rule in Eq. (1) implies that the potential supports a bound state at each energy for which the function $n(E)$ is an integer. The level density $\rho(E)$ is defined such that $\rho(E) d E$ is the (expected) number of energy levels in the energy interval $(E, E+d E)$.

The behavior of $n(E)$ near threshold, $E \rightarrow 0$, depends sensitively on the behavior of the potential $V(r)$ at large distances, $r \rightarrow \infty$. Potentials with an attractive tail falling off as

$$
V(r) \stackrel{r \rightarrow \infty}{\sim}-\frac{\hbar^{2}}{2 \mathcal{M}} \frac{\beta^{m-2}}{r^{m}}
$$

support an infinite number of bound states if the (not necessarily integer) power $m$ lies in the range $0<m<2$. Semiclassical approximations become increasingly accurate for $E$ $\rightarrow 0$ in this case [1], so the near-threshold quantization rule and level density can accurately be derived via straightforward WKB methods. This leads to a simple generalization of the well-known formula applicable for Coulombic potentials $(m=1)$, namely,

$$
n=A+\frac{F(m)}{\pi}(\kappa \beta)^{1-2 / m} \text { with } \quad F(m)=\frac{\sqrt{\pi}}{2 m} \frac{\Gamma\left(\frac{1}{m}-\frac{1}{2}\right)}{\Gamma\left(\frac{1}{m}+1\right)} \text {, }
$$

i.e., $n=A+B(-E)^{(1 / 2)-(1 / m)}$. The associated level density is characterized by a singularity proportional to $(-E)^{-(1 / 2)-(1 / m)}$ near threshold. Note that the coefficient of the leading energy-dependent term in Eq. (3) is completely determined by the power $m$ and the strength parameter $\beta$ of the leading $1 / r^{m}$ term in the potential tail. The constant $A$ on the right-hand side of Eq. (3) depends on the potential at shorter distances, where it may deviate from the asymptotic $1 / r^{m}$ form; for Coulombic potentials it is usually called the "quantum defect" [1].

For potentials falling off faster than $1 / r^{2}$, there is at most a finite number of bound states and conventional WKB quantization fails near threshold, but for a sufficiently deep well there may be a region of moderate $r$ values in the well where WKB wave functions are very accurate solutions of the Schrödinger equation for near-threshold energies. There has recently been considerable progress in the understanding of near-threshold properties in such cases [2-9].

For a potential tail falling off asymptotically as $1 / r^{2}$,

$$
V(r) \stackrel{r \rightarrow \infty}{\sim} \frac{\hbar^{2}}{2 \mathcal{M}} \frac{\gamma}{r^{2}},
$$

the near-threshold properties depend sensitively on the strength parameter $\gamma$. An attractive tail of sufficient strength, $\gamma<-1 / 4$, supports an infinite "dipole series" of bound states, the energies of which behave as 


$$
E_{n} \stackrel{n \rightarrow \infty}{=}-A \exp \left(-\frac{2 \pi n}{\sqrt{\gamma+1 / 4}}\right)
$$

towards threshold. Such attractive $1 / r^{2}$ potential tails can occur through a dipole-monopole interaction and its coupling to the orbital angular momentum, as, e.g., in the interaction of an electron and an excited hydrogen atom [10-12]. The ratio $E_{n} / E_{n+1}$ of successive energy eigenvalues in such a dipole series depends only on the strength parameter $\gamma$ of the $1 / r^{2}$ term and is simply $\exp (2 \pi / \sqrt{\gamma+1 / 4})$, but the absolute values of the energies as determined by the constant $A$ in Eq. (5) depend on the nature of the potential at short distances, where it necessarily deviates from the $1 / r^{2}$ behavior. Explicit expressions for $A$ have recently been derived for the case that the potential tail consists of a sufficiently strong attractive $1 / r^{2}$ term together with a shorter-ranged contribution proportional to $1 / r^{m}[13]$.

In Ref. [13] we discussed the near-threshold quantization rule for strongly attractive inverse square tails, but we did not do so for potential wells with weakly attractive or repulsive inverse-square tails, which support at most a finite number of bound states. It is the aim of this paper to close this gap and so to arrive at a comprehensive overview over the nature of near-threshold quantization rules and level densities for any strength of the $1 / r^{2}$ term in the potential tail.

In Sec. II we study weak (attractive or repulsive) $1 / r^{2}$ tails for which the strength parameter $\gamma$ defined according to Eq. (4) lies between $-1 / 4$ and $+3 / 4$. In Sec. III we focus on the limiting case $\gamma=-1 / 4$ at which the inverse-square potential ceases to support an infinite dipole series of bound states. This case is of very general importance since it corresponds to the $s$-wave centrifugal potential in the radial Schrödinger equation for a two-dimensional system. A summary also mentioning the connection to more strongly repulsive or attractive tails is given in Sec. IV.

\section{WEAK INVERSE-SQUARE TAILS}

We consider potential tails of the following form:

$$
V(r)=\frac{\hbar^{2}}{2 \mathcal{M}}\left(\frac{\gamma}{r^{2}}-\frac{\beta^{m-2}}{r^{m}}\right), \quad \beta>0, \quad-\frac{1}{4}<\gamma<\frac{3}{4}
$$

The term proportional to $1 / r^{m}$ is attractive and, in this section and the next, the (not necessarily integer) power $m$ is assumed to be greater than 2. This guarantees that the WKB approximation becomes increasingly accurate towards small $r$ values, so in a potential well obtained by supplementing Eq. (6) with an appropriate short-ranged repulsive contribution there can be a range of moderate $r$ values in the well where the WKB approximation is accurate for near-threshold energies; beyond this WKB region the potential is assumed to be given by Eq. (6). Such a potential well can support a large but at most finite number of bound states.

At energy $E=-\hbar^{2} \kappa^{2} /(2 \mathcal{M})$ the Schrödinger equation beyond the $\mathrm{WKB}$ region reads

$$
\left(\frac{d^{2}}{d r^{2}}-\frac{\gamma}{r^{2}}+\frac{\beta^{m-2}}{r^{m}}-\kappa^{2}\right) \psi(r)=0
$$

Asymptotically, $r \rightarrow \infty$, this reduces to

$$
\left(\frac{d^{2}}{d r^{2}}-\frac{\gamma}{r^{2}}-\kappa^{2}\right) R=0
$$

which posesses analytical solutions depending only on $\kappa r$. The solution which decays as required for a bound state is

$$
R(\kappa r)=i e^{i \mu \pi / 2} \sqrt{\kappa r} H_{\mu}^{(1)}(i \kappa r) \stackrel{\kappa r \rightarrow \infty}{\sim} \sqrt{\frac{2}{\pi}} \exp (-\kappa r),
$$

where $H_{\mu}^{(1)}$ is the Hankel function as defined in [14], and its order $\mu$ is related to $\gamma$ by

$$
\mu=\sqrt{\gamma+1 / 4}
$$

The range of strength parameters $\gamma$ defining the "weak" (attractive or repulsive) inverse-square term in Eq. (6) corresponds to the following range of values of $\mu$ :

$$
0<\mu<1
$$

For small arguments $\kappa r$ Eq. (9) becomes

$$
\begin{aligned}
R(\kappa r)= & \frac{\sqrt{2}}{\sin (\pi \mu)}\left[\frac{(\kappa r / 2)^{(1 / 2)-\mu}}{\Gamma(1-\mu)}-\frac{(\kappa r / 2)^{(1 / 2)+\mu}}{\Gamma(1+\mu)}\right] \\
& \times\left[1+O\left((\kappa r)^{2}\right)\right] .
\end{aligned}
$$

At intermediate values of $r$ the potential is deep and we neglect the energy term in the Schrödinger equation (7),

$$
\left(\frac{d^{2}}{d r^{2}}-\frac{\gamma}{r^{2}}+\frac{\beta^{m-2}}{r^{m}}\right) M(r)=0 .
$$

Two linearly independent solutions of Eq. (13) are

$$
M_{ \pm}(r)=\sqrt{r} J_{ \pm \nu}(z), \quad z=\frac{2}{m-2}\left(\frac{\beta}{r}\right)^{(m-2) / 2}
$$

where the abbreviation $\nu$ stands for

$$
\nu=\frac{2 \mu}{m-2}=\frac{2}{m-2} \sqrt{\gamma+\frac{1}{4}}
$$

and $J_{ \pm \nu}$ are the ordinary Bessel functions of order $\nu$ and $-\nu$, respectively [14]. The most general solution of Eq. (13) is a linear superposition,

$$
M(r)=A_{+} M_{+}(r)+A_{-} M_{-}(r) .
$$

For large $r$ corresponding to small $z$, the wave functions (14) behave as

$$
M_{ \pm}(r) \stackrel{r \rightarrow \infty}{=} \frac{\beta^{ \pm \mu} r^{(1 / 2) \mp \mu}}{\Gamma(1 \pm \nu)(m-2)^{ \pm \nu}}\left[1+O\left(\left(\frac{\beta}{r}\right)^{m-2}\right)\right]
$$


The leading $r$ dependence of $M_{+}$and $M_{-}$corresponds to the $r$ dependence of the leading near-threshold terms of Eq. (12), and, for a given (small) value of $\kappa$ we can determine the ratio of the coefficients $A_{+}$and $A_{-}$in Eq. (16) by requiring the ratio of the coefficients of $r^{(1 / 2)-\mu}$ and $r^{(1 / 2)+\mu}$ in Eq. (16) to be the same as in Eq. (12). This yields

$$
\begin{aligned}
\frac{A_{-}}{A_{+}} & =-\frac{(\kappa \beta / 2)^{2 \mu}}{(m-2)^{2 \nu}} \frac{\Gamma(1-\nu) \Gamma(1-\mu)}{\Gamma(1+\nu) \Gamma(1+\mu)} \\
& =-\frac{\pi^{2}(\kappa \beta / 2)^{2 \mu}}{\sin (\pi \mu) \sin (\pi \nu)(m-2)^{2 \nu} \mu \nu[\Gamma(\mu) \Gamma(\nu)]^{2}} .
\end{aligned}
$$

For certain values of $m$ in the range $2<m \leqslant 2+2 \mu$, the parameter $\nu=2 \mu /(m-2)$ can be an integer, so the gamma function $\Gamma(1-\nu)$ in Eq. (18) becomes singular, as expressed by the factor $\sin (\pi \nu)$ in the denominator in the second line. The reason for this singularity is the linear dependence of the two Bessel functions in Eq. (14) when $\nu$ is exactly an integer; one of the solutions $M_{+}$or $M_{-}$should be replaced, e.g., by $\sqrt{r} Y_{\nu}(z)$ in this case. In the derivation below, however, the results obtained for the near-threshold quantization rule and the level density for noninteger $\nu$ are free of singularities and can be continued through possible integer values, see, e.g., Eq. (33).

The wave function (9) with the near-threshold behavior (12) is an accurate solution of the Schrödinger equation (7) as long as the shorter-ranged term $\beta^{m-2} / r^{m}$ in the potential is negiligible compared to the longer-ranged term $\gamma / r^{2}$,

$$
r \gg \frac{\beta}{|\gamma|^{1 /(m-2)}} .
$$

The wave function (16) with the asymptotic behavior given by Eq. (17) is an accurate solution of the Schrödinger equation (7) as long as the energy term $\kappa^{2}$ is negiligible compared to the longer-ranged term in the potential,

$$
r \ll \frac{\sqrt{|\gamma|}}{\kappa} .
$$

We can match the wave functions (16) and (9), respectively (17) and (12) if there is a region of $r$ values, where the two conditions (19) and (20) are fulfilled simultaneously. Such a region exists when

$$
\kappa \beta \ll|\gamma|^{m /(2 m-4)},
$$

i.e., as long as $\gamma \neq 0$, the matching procedure described above is well justified in the limit $\kappa \rightarrow 0$.

Towards smaller values of $r$, the argument $z$ of the Bessel functions in Eq. (14) becomes large, and their asymptotic expansion yields

$$
M_{ \pm}(r) \stackrel{z \rightarrow \infty}{=} \sqrt{\frac{2 r}{\pi z}} \cos \left(z \mp \frac{\nu}{2} \pi-\frac{\pi}{4}\right),
$$

so the superposition (16) becomes

$$
\begin{aligned}
M_{\kappa}(r) \stackrel{z \rightarrow \infty}{=} & \sqrt{\frac{2 r}{\pi z}}\left[A_{+} \cos \left(z-\frac{\nu}{2} \pi-\frac{\pi}{4}\right)\right. \\
& \left.+A_{-} \cos \left(z+\frac{\nu}{2} \pi-\frac{\pi}{4}\right)\right] \\
& \propto \sqrt{\frac{r}{z}} \cos \left(z-\frac{\pi}{4}+\delta\right),
\end{aligned}
$$

where $\delta$ is a phase angle given by

$$
\tan \delta=-\frac{1-A_{-} / A_{+}}{1+A_{-} / A_{+}} \tan \left(\frac{\pi \nu}{2}\right) .
$$

We have included a subscript label $\kappa$ on the wave function (23) in order to emphasize the dependence on $\kappa$, which enters via the $\kappa$-dependent ratio (18) obtained by matching to the asymptotic solution $R(\kappa r)$.

The $r$ dependence of both amplitude and phase of the wave function (23) is that of the WKB wave function,

$$
\psi_{\mathrm{WKB}}(r) \propto \frac{1}{\sqrt{p(r)}} \cos \left(\frac{1}{\hbar} \int_{r_{\text {in }}}^{r} p\left(r^{\prime}\right) d r^{\prime}-\frac{\phi_{\text {in }}}{2}\right),
$$

at threshold, $E=0$, when the potential near $r$ is given by the shorter-ranged term in Eq. (6) alone. In Eq. (25), $p(r)$ $=\sqrt{2 \mathcal{M}(E-V(r))}$ is the local classical momentum and $\phi_{\text {in }}$ is the reflection phase at the inner classical turning point $[13,15]$, which is assumed to be a smooth function of energy, i.e., of $\kappa^{2}$, near threshold. At $E=0$ we have $p(r)=p_{0}(r)$ $=\sqrt{-2 \mathcal{M V} V(r)}$, and $p_{0}(r)=\hbar \beta^{(m-2) / 2} / r^{m / 2}$ when the potential is dominated by the term proportional to $1 / r^{m}$. Hence

$$
\frac{1}{\hbar} \int_{r_{\mathrm{in}}}^{r} p_{0}\left(r^{\prime}\right) d r^{\prime}=\text { const }-z,
$$

with $z$ as defined in Eq. (14).

If the WKB region overlaps with a range of $r$ values where the potential is dominated by the $1 / r^{m}$ term, then the quantization condition can be formulated by matching the wave functions (25) and (23) in this range of overlap. We expect the WKB wave function here to depend smoothly (analytically) on the energy $E$, so, to order less than $\kappa^{2}$, we can assume $E=0$ in Eq. (25). Equating the cosines in Eqs. (25) and (23) leads to the quantization condition

$$
\frac{1}{\hbar} \int_{r_{\mathrm{in}}}^{r} p_{0}\left(r^{\prime}\right) d r^{\prime}-\frac{\phi_{\text {in }}}{2}=n \pi-z+\frac{\pi}{4}-\delta+O(E) .
$$

The subscript zero on the local momentum $p$ indicates that the action integral on the left-hand side is to be taken at threshold, $E=0$. In the range of overlap, where the WKB approximation is accurate and the potential is dominated by the $1 / r^{m}$ term, the expression 


$$
I_{0}=\frac{1}{\hbar} \int_{r_{\text {in }}}^{r} p_{0}\left(r^{\prime}\right) d r^{\prime}+z-\frac{\phi_{\text {in }}}{2}-\frac{\pi}{4}
$$

is independent of $r$. Using Eq. (24) we can thus rewrite the quantization condition (27) as

$$
\begin{aligned}
n \pi & =I_{0}+\delta+O(E) \\
& =I_{0}-\arctan \left[\tan \left(\frac{\pi \nu}{2}\right) \frac{1-A_{-} / A_{+}}{1+A_{-} / A_{+}}\right]+O(E) .
\end{aligned}
$$

With the help of the identity,

$$
\begin{aligned}
& \arctan \left[\tan \left(\frac{\pi \nu}{2}\right) \frac{1-A_{-} / A_{+}}{1+A_{-} / A_{+}}\right] \\
& =\frac{\pi \nu}{2}-\arctan \left[\frac{\left(A_{-} / A_{+}\right) \sin (\pi \nu)}{1+\left(A_{-} / A_{+}\right) \cos (\pi \nu)}\right],
\end{aligned}
$$

this is seen to correspond, to leading orders in $\kappa$, to

$$
\begin{aligned}
n= & \frac{I_{0}}{\pi}-\frac{\nu}{2}-\frac{\pi(\kappa \beta / 2)^{2 \mu}}{\sin (\pi \mu)(m-2)^{2 \nu} \mu \nu[\Gamma(\mu) \Gamma(\nu)]^{2}} \\
& +O\left((\kappa \beta)^{4 \mu}\right)+O\left(\kappa^{2}\right),
\end{aligned}
$$

where we have expressed the (small) quantity $A_{-} / A_{+}$in terms of $\kappa$ according to Eq. (18).

The quantization condition (31) has the form

$$
\begin{aligned}
& E \rightarrow 0 \\
& n \stackrel{=}{=} A-B(-E)^{\mu},
\end{aligned}
$$

and the correction to the right-hand side is of order $E$ if $2 \mu>1$ and of order $(-E)^{2 \mu}$ if $2 \mu<1$. The constant $A$ $=I_{0} / \pi-\nu / 2$ represents the threshold value of the quantum number that determines the total number of bound states supported by the potential. The constant $B$ is a "tail parameter" that depends only on the tail of the potential beyond the WKB region. For the potential tail (6) we have

$$
B=\frac{\pi\left(\mathcal{M} \beta^{2} /\left(2 \hbar^{2}\right)\right)^{\mu}}{\sin (\pi \mu)(m-2)^{2 \nu} \mu \nu[\Gamma(\mu) \Gamma(\nu)]^{2}},
$$

as derived above. The explicit value of $B$ depends on the further term that complements the $\gamma / r^{2}$ contribution in the potential tail and guarantees that there is a region of $r$ values in the well where WKB wave functions become accurate solutions of the Schrödinger equation at near-threshold energies. The power $\mu=\sqrt{\gamma+1 / 4}$ in Eq. (32) depends only on the strength $\gamma$ of the $1 / r^{2}$ term, and not on the properties of the shorter-ranged contribution to the potential tail.

The near-threshold level density follows from Eq. (32),

$$
\rho(E)=\frac{d n^{E \rightarrow 0}}{d E} \sim B \mu|E|^{\mu-1}
$$

Since $0<\mu<1$, the level density becomes infinite near threshold, even though the total number of bound states remains finite.

In Ref. [13] we calculated the leading near-threshold behavior of the transmission probability $P_{\mathrm{T}}$ through a centrifugal barrier consisting of two terms as in Eq. (6). For positive energies $E=\hbar^{2} k^{2} /(2 \mathcal{M})$, the leading energy dependence of the transmission probability is,

$$
P_{\mathrm{T}} \stackrel{E \rightarrow 0}{=} \frac{4 \pi^{2}(k \beta / 2)^{2 \mu}}{(m-2)^{2} \mu \nu[\Gamma(\mu) \Gamma(\nu)]^{2}} .
$$

As pointed out in [13], this result holds both for repulsive inverse-square terms $(\gamma>0)$ and for weakly attractive terms, $-1 / 4<\gamma<0$. The leading energy dependence in Eq. (35) is $P_{\mathrm{T}} \propto k^{2 \mu} \propto E^{\mu}$. For three-dimensional systems, the centrifugal potential for the angular momentum quantum number $l$ corresponds to $\mu=l+1 / 2$, so the proportionality to $E^{\mu}$ simply expresses Wigner's threshold law. (Note that Wigner's threshold law also works for weakly attractive inverse-square potentials formally corresponding to $-\frac{1}{2}<l<0$.) For potential wells with weak inverse-square tails, the leading energy dependence of the near-threshold quantization rule (31) is proportionality to $\kappa^{2 \mu} \propto(-E)^{\mu}$, and the coefficient of this term is closely related to the corresponding coefficient in Eq. (35). Comparing Eqs. (31) and (35) shows that the coefficient of $k^{2 \mu}$ in the expression (35) for the transmission probability through the potential tail is just $4 \pi \sin (\pi \mu)$ times the coefficient of $\kappa^{2 \mu}$ in the near-threshold quantization rule (31). This factor depends only on the strength $\gamma$ of the $1 / r^{2}$ term and not on the properties of the shorter-ranged contribution to the potential tail.

For a potential well with a tail vanishing faster than $1 / r^{2}$ asymptotically, it has been shown previously [4-9] that the near-threshold quantization rule has the form

$$
\stackrel{E \rightarrow 0}{n}=A-B \sqrt{-E}+O(E)
$$

where $A$ and $B$ are constants, and that the leading behavior of the transmission probability through the potential tail is $[7,16]$

$$
P_{\mathrm{T}}=4 \pi B \sqrt{E}
$$

where the constant $B$ is the same in both Eqs. (36) and (37) and depends only on the potential tail beyond the WKB region. These results correspond exactly to those derived above, Eqs. (31)-(35), for the special case of vanishing strength of the inverse-square term, $\gamma=0, \mu=1 / 2$. The expression (33) is equivalent to the formula (2) in Ref. [8] corresponding to Eq. (9) in Ref. [4], and Eq. (35) above corresponds to Eqs. (35) and (36) in Ref. [16] when $\mu$ $=1 / 2$. (Generalizations to potential tails with significant deviations from the homogeneous form proportional to $1 / r^{m}$ beyond the WKB region are discussed in Ref. [7].)

The derivations in this section have been limited to strength parameters in the range $-\frac{1}{4}<\gamma<\frac{3}{4}$ corresponding to "weak" inverse-square tails. The upper end of this inter- 
val, $\gamma=3 / 4$, corresponds to $\mu=1$, so the leading terms proportional to $(-E)^{\mu}$ in Eqs. (31) and (32) are of the same order, viz., $O(E)$, as terms neglected by replacing the action integral in Eq. (27) by its value at threshold. For repulsive inverse-square potentials with $\gamma=3 / 4$ or larger, we can expect the near-threshold quantization rule to be of the form

$$
\stackrel{E \rightarrow 0}{n} \stackrel{\text { const }}{ }+O(E),
$$

so the resulting level density $\rho=d n / d E$ remains finite at threshold.

The lower end of the range of strength parameters discussed in this section is $\gamma=-1 / 4$ corresponding to $\mu=0$. This special case is the subject of the next section.

\section{THE SPECIAL CASE $\gamma=-1 / 4$}

The near-threshold quantization rule (32) and the formula (34) for the near-threshold level density become meaningless for $\gamma=-1 / 4$ corresponding to $\mu=0$. An inverse-square potential behaving asymptotically as $-\frac{1}{4} \hbar^{2} /\left(2 \mathcal{M r}{ }^{2}\right)$ represents the limit for which the potential will no longer support an infinite dipole series of bound states. This case is of central importance for two-dimensional problems, where the centrifugal potential in the radial Schrödinger equation is $\left(l^{2}-1 / 4\right) \hbar^{2} /\left(2 \mathcal{M} r^{2}\right)(l=0,1,2, \ldots), \quad$ rather than $l(l$ $+1) \hbar^{2} /\left(2 \mathcal{M} r^{2}\right)$ applicable in the three-dimensional case. For $s$ waves $(l=0)$ in two dimensions, the centrifugal potential is attractive, and the strength parameter according to the definition (4) is precisely $\gamma=-1 / 4$.

As in Sec. II, we assume that there is a region of moderate $r$ values where the WKB approximation is accurate at nearthreshold energies, and that the potential is described by the following two terms beyond this WKB region:

$$
V(r)=-\frac{\hbar^{2}}{2 \mathcal{M}}\left(\frac{1 / 4}{r^{2}}+\frac{\beta^{m-2}}{r^{m}}\right), \quad m>2 .
$$

At energy $E=-\hbar^{2} \kappa^{2} /(2 \mathcal{M})$ the Schrödinger equation beyond the WKB region is given by Eq. (7) with $\gamma=-1 / 4$. Asymptotically, $r \rightarrow \infty$, this reduces to Eq. (8), and for $\gamma$ $=-1 / 4$ the solution which decays as required for a bound state is

$$
R(\kappa r)=i \sqrt{\kappa r} H_{0}^{(1)}(\mathrm{i} \kappa r) \stackrel{\kappa r \rightarrow \infty}{\sim} \sqrt{\frac{2}{\pi}} \exp (-\kappa r),
$$

where $H_{0}^{(1)}$ is the Hankel function of order zero. For small arguments we now have

$$
R(\kappa r) \stackrel{\kappa r \rightarrow 0}{\sim}-\frac{2}{\pi}\left[\ln \left(\frac{\kappa r}{2}\right)+\gamma_{\mathrm{E}}\right] \sqrt{\kappa r}\left[1+O\left((\kappa r)^{2}\right)\right],
$$

where $\gamma_{\mathrm{E}}$ is Euler's constant [14].

At intermediate values of $r$ the potential is deep and we neglect the energy term in the Schrödinger equation (7) and consider Eq. (13). For $\gamma=-1 / 4$, this equation is solved [14] by functions of the form $\sqrt{r} \mathcal{C}_{0}(z)$, where $\mathcal{C}_{0}$ is (any) Bessel function of order zero, and the argument $z$ is as already defined in Eq. (14). The most general solution $M(r)$ of Eq. (13) (with $\gamma=-1 / 4$ ) can be expressed as a linear superposition of two linearly independent solutions generated, e.g., by the Bessel functions $J_{0}(z)$ and $Y_{0}(z)$,

$$
M(r)=\sqrt{r}\left[A_{J} J_{0}(z)+A_{Y} Y_{0}(z)\right] .
$$

For large values of $r$ corresponding to small values of $z$ this solution behaves as

$$
M(r) \stackrel{r \rightarrow \infty}{\sim} \sqrt{r}\left\{A_{J}+\frac{2 A_{Y}}{\pi}\left[\ln \left(\frac{z}{2}\right)+\gamma_{\mathrm{E}}\right]\right\},
$$

which contains the same leading $r$ dependence $[\propto \sqrt{r}$ (const $-\ln r)]$ as the near-threshold form (41) of the solution of Eq. (8). Matching the two leading terms of $M(r)$ and $R(\kappa r)$ leads to the following result for the ratio of the coefficients in the expression (42) for $M(r)$ :

$$
\frac{A_{J}}{A_{Y}}=-\frac{1}{\pi}\left[m \gamma_{\mathrm{E}}+2 \ln \left(\frac{(\kappa \beta / 2)^{(m-2) / 2}}{m-2}\right)\right] .
$$

As in Sec. II, this matching is justified if there is a region of $r$ values where both Schrödinger equations (8) and (13) are simultaneously accurate approximations of the full Schrödinger equation (7), leading to the condition (21) (with $\gamma=-1 / 4$ ), which is increasingly well fulfilled towards threshold.

Towards smaller values of $r$ (larger $z$ ), the asymptotic expansion of the Bessel functions in Eq. (42) yields

$$
\begin{aligned}
M_{\kappa}(r) \stackrel{z \rightarrow \infty}{ } & \propto \sqrt{\frac{r}{z}}\left[\frac{A_{J}}{A_{Y}} \cos \left(z-\frac{\pi}{4}\right)+\sin \left(z-\frac{\pi}{4}\right)\right] \\
& \propto \sqrt{\frac{r}{z}} \cos \left(z-\frac{\pi}{4}+\delta\right),
\end{aligned}
$$

where the phase angle $\delta$ is now given by

$$
\cot \delta=\frac{1}{\pi}\left[m \gamma_{\mathrm{E}}+2 \ln \left(\frac{(\kappa \beta / 2)^{(m-2) / 2}}{m-2}\right)\right] .
$$

In the region of overlap, where the WKB approximation is accurate and the potential is dominated by the $1 / r^{m}$ term, matching the right-hand side of Eq. (45) to the WKB wave function (25) now yields

$$
n \pi=I_{0}+\delta=I_{0}+\operatorname{arccot}\left\{\frac{1}{\pi}\left[m \gamma_{\mathrm{E}}+2 \ln \left(\frac{(\kappa \beta / 2)^{(m-2) / 2}}{m-2}\right)\right]\right\},
$$

where $I_{0}$ is again given by Eq. (28) and does not depend on the value of $r$, as long as $r$ is chosen in the region where the WKB approximation is accurate and the potential is dominated by the $1 / r^{m}$ term. Near threshold, $\kappa \rightarrow 0$, the argument of the arcus-cotangent on the right-hand side of Eq. (47) 
TABLE I. Summary of near-threshold quantization rules for potential wells with tails given by Eq. (51). For $m>2, \gamma>-1 / 4$ the table also contains the leading near-threshold behavior of the transmission probability $P_{\mathrm{T}}$ through the tail. The symbols $A$ and $B$ refer to constants appropriate to the respective cases. Whereas $A$ generally depends on properties of the whole potential well, the parameter $B$ depends only on the properties of the tail (51). The last column lists equations containing explicit expressions for the constants $A$ and/or $B$ in the respective cases.

\begin{tabular}{lcc}
\hline \hline $0<m<2$, any $\gamma$ & $\begin{array}{l}E \rightarrow 0 \\
n=A+B(-E)^{(1 / 2)-(1 / m)}\end{array}$ \\
\hline
\end{tabular}

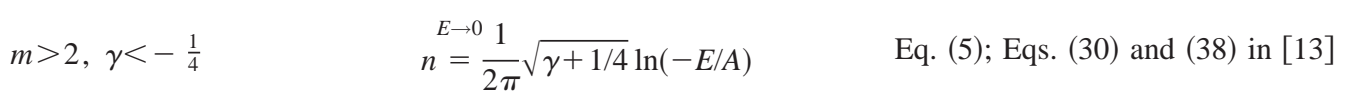
$m>2, \gamma=-\frac{1}{4}$
$E \rightarrow 0$
$n=A+2 /[(m-2) \ln (-E / B)]$
Eqs. (48) and (49)
$m>2,-\frac{1}{4}<\gamma<\frac{3}{4}$
$E \rightarrow 0$
$n=A-B(-E)^{\sqrt{\gamma+1 / 4}}$
Eqs. (31) and (33)
$\stackrel{E \rightarrow 0}{P_{\mathrm{T}}}=4 \pi B \sin (\pi \sqrt{\gamma+1 / 4}) E^{\sqrt{\gamma+1 / 4}}$
Eqs. (35) and (33); Eq. (57) in [13]

$m>2, \gamma \geqslant \frac{3}{4} \quad \begin{gathered}E \rightarrow 0 \\ n=A+O(E) \\ E \rightarrow 0 \\ P_{\mathrm{T}} \propto E^{\sqrt{\gamma+1 / 4}}\end{gathered}$

Eq. (35); Eq. (57) in [13]

becomes a large (negative) number, so the leading contribution to the near-threshold quantization rule is

$$
\begin{aligned}
n & =\frac{\kappa \rightarrow 0}{\pi} I_{0} \\
& =\frac{1}{m \gamma_{\mathrm{E}}+2 \ln \left(\frac{(\kappa \beta / 2)^{(m-2) / 2}}{m-2}\right)} \\
\pi & \frac{1}{a_{0}+(m-2) \ln (\kappa \beta)},
\end{aligned}
$$

with $a_{0}=m \gamma_{\mathrm{E}}-2 \ln (m-2)-(m-2) \ln 2$. In terms of energy,

$$
\begin{aligned}
& n \stackrel{E \rightarrow 0}{=} \frac{I_{0}}{\pi}+\frac{1}{a_{0}+\frac{m-2}{2} \ln (-E / B)} \\
& =\frac{I_{0}}{\pi}+\frac{2 /(m-2)}{\ln (-E / B)}+O\left(\frac{1}{[\ln (-E / B)]^{2}}\right),
\end{aligned}
$$

with $B=\hbar^{2} /\left(2 \mathcal{M} \beta^{2}\right)$. The leading contribution to the nearthreshold level density is

$$
\begin{aligned}
\rho(E) & =\frac{d n^{E \rightarrow 0}}{d E}=\frac{m-2}{2(-E)} \frac{1}{\left(a_{0}+\frac{m-2}{2} \ln (-E / B)\right)^{2}} \\
& =\frac{2 /(m-2)}{(-E)[\ln (-E / B)]^{2}}+O\left(\frac{1}{(-E)[\ln (-E / B)]^{3}}\right) .
\end{aligned}
$$

\section{SUMMARY AND DISCUSSION}

We have studied potential wells with tails behaving as

$$
V(r)=\frac{\hbar^{2}}{2 \mathcal{M}}\left(\frac{\gamma}{r^{2}}-\frac{\beta^{m-2}}{r^{m}}\right)
$$

For $m>2$ the near-threshold properties depend crucially on the strength parameter $\gamma$ of the inverse-square term. For a weak inverse-square term with $-\frac{1}{4}<\gamma<\frac{3}{4}$, the nearthreshold quantization rule has the general form,

$$
\begin{aligned}
& E \rightarrow 0 \\
& n=A-B(-E)^{\sqrt{\gamma+1 / 4}}+O(E),
\end{aligned}
$$

where $A$ and $B$ are constants. The related level density $\rho$ $=d n / d E$ is characterized by a singularity proportional to $(-E)^{\sqrt{\gamma+1 / 4}-1}$ near threshold; it becomes infinite, even 
though the number of bound states in the well is finite. For positive energies just above threshold, the probability for transmission through the potential tail is, to leading order, $4 \pi B \sin (\pi \sqrt{\gamma+1 / 4}) E^{\sqrt{\gamma+1 / 4}}$. The same constant $B$ appears in the quantization rule and the transmission probability, and it depends only on the tail of the potential beyond the region where the WKB approximation is accurate. Results derived previously for potential tails falling off faster than $1 / r^{2}$ fit into this scheme if we put $\gamma=0$, corresponding to vanishing strength of the inverse-square term.

For repulsive inverse-square terms beyond the range referred to above, $\gamma \geqslant 3 / 4$, the leading energy dependence of $n(E)$ near threshold is of order $O(E)$, and the level density remains finite. This means that for a potential well with a repulsive inverse-square tail at least as strong as the $p$-wave centrifugal term in two-dimensional systems, there is no singular contribution to the level density near threshold. The transmission probability through the barrier is still given by Eq. (35) and obeys Wigner's threshold law.

For attractive inverse-square tails with $\gamma=-1 / 4$, the leading energy dependence of $n(E)$ near threshold is proportional to $1 / \ln (-E)$. For more strongly attractive inversesquare tails it is proportional to $\ln (-E)$, and the potential well supports an infinite dipole series of bound states, as described by Eq. (5). This behavior connects to the case of longer-ranged potential tails which fall off slower than $1 / r^{2}$, see Eq. (3).

These results are summarized in Table I. It covers all potential tails of the form (51) with arbitrary values of $\gamma, m$ $>0$, and $\beta>0$.
[1] H. Friedrich, Theoretical Atomic Physics, 2nd ed. (SpringerVerlag, Berlin, 1998).

[2] J. Trost, C. Eltschka, and H. Friedrich, J. Phys. B 31, 361 (1998).

[3] C. Boisseau, E. Audouard, and J. Vigué, Europhys. Lett. 41, 349 (1998).

[4] J. Trost, C. Eltschka, and H. Friedrich, Europhys. Lett. 43, 230 (1998).

[5] V.V. Flambaum, G.F. Gribakin, and C. Harabati, Phys. Rev. A 59, 1998 (1999).

[6] C. Boisseau, E. Audouard, J. Vigué, and V.V. Flambaum, Eur. Phys. J. D 12, 199 (2000).

[7] C. Eltschka, M.J. Moritz, and H. Friedrich, J. Phys. B 33, 4033 (2000), special issue on coherent matter waves, edited by $\mathrm{K}$. Burnett.

[8] C. Eltschka, H. Friedrich, and M.J. Moritz, Phys. Rev. Lett. 86,
2693 (2001).

[9] C. Boisseau, E. Audouard, and J. Vigué, Phys. Rev. Lett. 86, 2694 (2001).

[10] T. Purr, H. Friedrich, and A.T. Stelbovics, Phys. Rev. A 57, 308 (1998).

[11] E. Lindroth, A. Bürgers, and N. Brandefelt, Phys. Rev. A 57, R685 (1998).

[12] T. Purr and H. Friedrich, Phys. Rev. A 57, 4279 (1998).

[13] M.J. Moritz, C. Eltschka, and H. Friedrich, Phys. Rev. A 63, 042102 (2001).

[14] Handbook of Mathematical Functions, edited by M. Abramowitz and I.A. Stegun (Dover, New York, 1972).

[15] H. Friedrich and J. Trost, Phys. Rev. Lett. 76, 4869 (1996); Phys. Rev. A 54, 1136 (1996).

[16] R. Côté, H. Friedrich, and J. Trost, Phys. Rev. A 56, 1781 (1997). 